\title{
The Evolution and Ecology of Resistance in Cancer Therapy
}

\author{
Robert Gatenby and Joel Brown \\ Cancer Biology and Evolution Program, Moffitt Cancer Center, Tampa, Florida 33612 \\ Correspondence: robert.gatenby@moffitt.org
}

Despite continuous deployment of new treatment strategies and agentc over many decades, most disseminated cancers remain fatal. Cancer cells, through the access $\mathrm{p}$ the vast information of human genome, have a remarkable capacity to deploy a hptive str tegies for even the most effective treatments. We note there are two critical sto in th linic 1 manifestation of treatment resistance. The first, which is widely invest ated equildoployment of a mechanism of resistance that usually involves increase ex molecular machinery necessary to eliminate the cytotoxic effect of treatm t. How er, emengence of a resistant phenotype is not in itself clinically signific.ht. hat is, rosistant cells affect patient outcomes only when they form a sufficiently popu. to to allow tumor progression and treatment failure. Importantly, prolifer , on of the resistant phenotype is by no means certain and, in fact, depends on complex arwiniar dynamics governed by the costs and benefits of the resistance mechanisms in the oxt the local environment and competing populations. Attempts to target mol fan machmery of resistance have had little clinical success largely because of the diversit, wi inne human genome-therapeutic interruption of one mechanism simply re arts $n$ its eplacement by an alternative. We explore an alternative strategy for overcon ng tre ament esistance that seeks to understand and exploit the critical evolutionary $d$ nat that govern proliferation of the resistant phenotypes. In general, this appro? has sho un that, although emergence of resistance mechanisms in cancer cells to every cur ant therapy is inevitable, proliferation of the resistant phenotypes is not and can b delayed an even prevented with sufficient understanding of the underlying ecoevolutir ary d hamics.

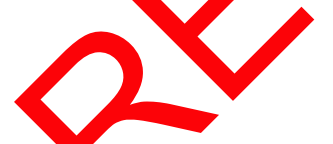

The rnamic cancer ecosystem, rich in tem-

porar and spatial diversity in environmental conditions and heritable cell phenotypes, is extraordinarily robust to therapeutic perturbations. In part, this is caused by cellular diversity, as spatial heterogeneity in the genotypic and phenotypic properties of tumor cells inevitably alter their response to applied treatments.
In part, this is also caused by temporal and spatial heterogeneity in the tumor environment, largely governed by variations in blood flow, which can alter both the delivery of a systemic agent and local concentrations of factors (e.g., oxygen) that may alter the efficacy of the treating agent. Finally, in part, the response and resistance of cancer cells is governed by their

Editors: Charles Swanton, Alberto Bardelli, Kornelia Polyak, Sohrab Shah, and Trevor A. Graham

Additional Perspectives on Cancer Evolution available at www.perspectivesinmedicine.org

Copyright (C) 2018 Cold Spring Harbor Laboratory Press; all rights reserved; doi: 10.1101/cshperspect.a033415

Cite this article as Cold Spring Harb Perspect Med 2018;8:a033415 
complex interactions with adjacent host cells, which can provide local sanctuaries that permit tumor cells to survive treatment that would ordinarily be lethal.

Despite the many advances in cancer therapy over the past few decades, evolution of resistance continues to limit their efficacy in reducing overall survival. Even after a response to therapy that is clinically complete, residual cancer cells adapt and the tumor almost inevitably returns. Although the evolution of resistance continues to be the central cause of death in most cancer patients, recent analysis found that evolutionary terms were included in fewer than one percent of manuscripts on cancer treatment outcomes; this has remained unchanged for 30 years.

In general, there are two approaches to investigating the dynamics of cancer cell resistance to therapy. The most common avenue of research focuses on identifying the specific molecular mechanisms of resistance. This is per haps best illustrated in the extensive lite ature on membrane extrusion pumps-P-glyc vroteins ( $\mathrm{PgP})$, for example - that us to a tively remove cytotoxic agents from the cur cell cytoplasm and send the $A \mathrm{ck}$ in $\sigma$ the environment. This approa has t eadvatage of identifying molecular med isms that can be targeted and, thu feated. Iowever, despite decades of reserch in 1l-recognized adaptive mechanism ach as the aultidrug resistance (MDR) ge ss, th $s$ approach has not led to clinically sifica ther py. In large part, this is the res $h$ of he ren ikable diversity of adaptive stra vi avamole through the human genome. Thus, ccessful therapeutic intervention in one resistance mechanism simply selects for a second available strategy.

Importantly, however, we note that the expression of a resistance mechanism does not by any means insure that the resistant population will rapidly proliferate leading to tumor progression. Thus, although the mechanisms of adaptation to the toxic effects of therapy are molecular, the clinical relevance of resistance is entirely dependent on proliferation of resistant populations - a process ultimately governed by Darwinian dynamics based on the phenotypic cost and benefit of the molecular mechanisms of resistance in the context of the local environment and competing cellular populations.

Here, we review treatment strategies that explicitly incorporate evolutionary principles to limit proliferation of resistant and prolong time to progression. Nearly all conventional cancer therapies assume that the maximum patient benefit is achieved through killing the greatest possible number of tumor cells. Thus, cancer treatments are typically administered at the maximum tolerated dose (MTD). Indeed, this principle is so universally accepted that phase 1 trials-necessary for clinical translation of any cancer drug - seen to define the MTD, which is then us in sub quent investigations. Althoug killing the $\mathrm{n}$ aximum number of tumor cell with ae gre est possible drug dose is intuiver, ppeali 8 , we propose that it is usually evolutio a y unwise. This reflects the principle n ted above-evolution of resistant molecular mac inery is virtually impossible to evade but proliferation of resistant populations is dependent on complex Darwinian dynamics. In fact, by applying intense Darwinian selection for resistant clones and elimination of all competing population, MTD cancer therapy actually "accelerates" proliferation of resistant populations (also well-known as an evolutionary phenomenon termed "competitive release") (Gatenby 2009; Renton et al. 2014). Thus, evolutionary responses of cancer cells to therapy becomes the proximate cause of death in most cancer patients and will most likely remain as such in want of basic changes in the tumor treatment paradigm.

The majority of conceptual models of tumor evolution during chemotherapy stress resistance acquired in a step-wise fashion through some mutation following the start of therapy (Anderson and Quaranta 2008). If resistance arises stochastically and before treatment there are no resistant cells present, then maximum dose-density therapy will reduce the number of cells that can acquire the mutation with the probability of resistance lessening. However, in most cases, the resistant cells or some level of resistance appears before chemotherapy starts (Easwaran et al. 2014).

The assumption that a "resistance mutation" is necessary for tumor adaptation under- 
estimates the vast information content, including xenobiotic pathways, that is available to cancer cells within the normal human genome (Gatenby 2009). That is, many, and probably most, resistance strategies only require an increased expression of one or more normal genes (PgP; see Schneider et al. 1989; Fletcher et al. 2010; Wind and Holen 2011). Therefore, rather than an "all or none" phenomenon, resistance can be graded among cells in a population and, importantly, change in the same cell over time as it acclimatizes to environmental stresses. For example, PgP is a hypoxia inducible factor (HIF) $1 \alpha$ client (Thews et al. 2011) and its expression often increases in hypoxic and acidic environments even without cytotoxins. In addition, numerous mechanisms of de novo therapy resistance have been identified. For example, in environmentally mediated drug resistance (EMDR), components of tumor mesenchyma protect cancer cells from what y ard otherwise be lethal concentrations of cyt toxic drugs (Meads et al. 2009; Moulder 2010). vimilarly, tumor cells in regions of hyt may protected as a result of increased ex res $10 \mathrm{ff}$ PgP, up-regulation survival at vays, ncreased mutagenesis, and decrea d dry deliv ry.

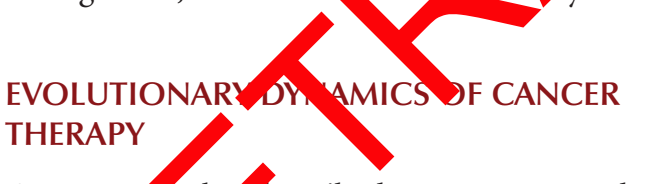

Cancers a bescribed as open complex adapti ysto ss. " pen" because they freely cop nuni ate wa their surroundings, "complex caus they contain multiple components, nd "adaptive" because each element can change over time and interact with other components in complicated, often nonlinear, ways. Based on their nonlinear dynamics, a critical attribute is that their dynamics can be nonintuitive and their response to a perturbation can yield unexpected and unintended consequences.

Traditional application of systemic therapy to cancer is largely based on an intuitively appealing premise because by killing the maximum number of cancer cells, patient benefit is achieved (Norton and Simon 1986; Rodrigues and de Arruda Mancera 2013). This assumption that more is better is so deeply ingrained, that the initial clinical trials (i.e., phase 1 trials), always seeks to establish the maximum tolerated dose for that drug. In other words, there is an implicit assumption that all cancer drugs must be administered at the maximum possible dose to find the MTD in the first phase. Generally, only out of concern for fatal toxicity was the maximum dose density of chemotherapy limited. An alternate approach is the "metronomic" strategy (Gnoni et al. 2015), which administers lower more frequent doses of therapy. This has been shown to be beneficial in lowering toxicity, while permi ang tor tor drug administration, and hibiting ngiogenesis by increasing tumo cell de h. H wever, the intent of moderp nera rem ins inducing the greatest posble 1 deat $^{\prime}>$ whether administered through maxim, r metronomic dosing.

Significant flaws in conventional assumption emerge when cancer therapy is viewed as an evolutionary process (Axtell and Arends 1990; Gatenby 2009; Gatenby et al. 2009a; Silva et al. 2012; Renton et al. 2014; Jansen et al. 2015; Kam et al. 2015). To be clear, when it is possible to have curative therapy, then the treatment strategy must be designed with that result in mind. However, in a palliative clinical setting (in which metastatic cancer patients nearly always die of their disease), treatment for cure is not only futile, it is, in fact, evolutionarily unsound. In destroying the entire population of sensitive cells, maximum dose therapy imposes intense selection for resistant phenotypes and, by eliminating all potential competitors, their proliferation is maximized (a well-known evolutionary phenomenon termed "competitive release") (Axtell and Arends 1990; Renton et al. 2014).

Interestingly, insight into these dynamics can be found in an unlikely source-pest and weed management (Oliveira et al. 2007; Neve et al. 2009). For decades, the application of high-dose pesticides was commonplace, but over time it became clear that this approach virtually never eradicated the pest and, in fact, promoted the rapid emergence of uncontrollable, resistant strains. Since 1968, the policy toward pest management by the U.S. De- 
partment of Agriculture (USDA) has been much more nuanced placing greater emphasis on the controlled application of pesticides. The goal is to prevent the emergence of pesticide resistance while maintaining a low and acceptable level of crop damage (Oliveira et al. 2007; Neve et al. 2009). Incorporation of temporal data sampling and Darwinian dynamics into the management of invasive species is now mandated by USDA policy. To assist agriculturalists in creating the best treatment strategies, computational models guide pest management, similar to those that we propose for adaptively managing advanced, incurable metastatic cancers.

\section{APPLICATION OF EVOLUTIONARY PRINCIPLES TO CANCER THERAPY RESISTANCE}

Evolutionary therapy usually zeroes in competition for space and resources a nong cancer populations, and in particular, $\mathrm{Da}$, inian interactions between resistant anreso tant populations. When a cancer the py to ts in cell death, this imposes st $\pi_{\mathrm{c}}$ elec on forces for adaptive strategies. $\mathrm{T}$ - size nd co pplexity of the human gen me actically assures the presence of ny rous po ntially adaptive pathways in which to roid cell death. Thus, although ta eting comb ations of pathways can contro hu an immunodeficiency virus (HIV) th o v nir protein-encoding genes, thi strate y has not as yet proved successful in tur the emergence of resistance in human ells.

If resistant populations cannot be prevented, then tumor control requires therapy designed to slow or stop the proliferation of resistance. Here, two basic fundamentals must be emphasized: first, the growth of the resistant population is subject to evolutionary forces and, therefore, can be controlled by altering its fitness or that of competing populations. Second, evolving populations can only adapt to local and current environmental selection forces; they can never anticipate the future. Importantly, because cancer therapists can anticipate the future and use the knowledge of these temporal dynamics to a key advantage by using evolution to inhibit the proliferation of resistant populations, they can allow treatment to change over time.

In evolutionary cancer treatment, a major component of Darwinian dynamics is the cost of resistance. To become resistant, typically through up-regulation of established molecular defense mechanisms, cancer cells must alter their observable properties. Expression, maintenance, and utilization of these molecular pathways require resources, which, in an environment of limited substrate, must be diverted from prolife ation nd invasion. These dynamics are per aps mos clear in up-regulation of xenob tic pa bay such as increased expressic of $\mathrm{Pa}$, also nown as multidrug resistance (M 1) (S meider et al. 1989; Fletcher et al. 2010; h and Holen 2011). PgP is a memb. ne transporter that effectively extrudes a larg number of intracellular substrates, including chemotherapies and, consequently, minimizes the efficacy of these compounds. PgP and most other membrane pumps hydrolyze two ATP for every transported molecule. Indeed, in experimental studies this operation cost (as well as "capital cost" for synthesis and maintenance of the pumps) can approach $50 \%$ of the cell's energy budget (Silva et al. 2012). In cell culture conditions with abundant resources, this may have minimum effect on cellular proliferation. However, under resource limitation (e.g., in vivo), cancer cells must trade-off resistance costs that permit survival with other essential functions such as proliferation, movement, and cell maintenance. This fitness cost can be inferred by the simple observation that the MDR phenotype is rare in pretherapy tumors and becomes common only following treatment (Wind and Holen 2011). Furthermore, the drug resistant phenotype is typically evolved through regular exposure to a cytotoxic drug and quickly lost when the drug is removed (Kam et al. 2015).

Thus, in the presence of a cytotoxic drug, survival benefits of resistance exceed the fixed and operating costs of the resistance mechanisms. But, in the absence of therapy, the cost of resistance becomes an ecological burden that can be exploited (Silva et al. 2015). 
The ecological cost of resistance is most apparent in traditional chemotherapy in which the mechanisms of resistance and their associated "construction and operation" costs are fairly clear. This is not as apparent in targeted therapies. However, Chmielecki et al. (2011) have shown a cost of resistance to tyrosine kinase inhibitors (TKIs).

\section{EXPLOITING THE COST OF RESISTANCE}

Theodosius Dobzhansky famously stated "Nothing in biology makes sense except in the light of evolution" (Ayala and Dobzhansky 1977). Yet, the typical cancer therapy is administered without regard to the evolutionary consequences. Drugs, doses, and schedules follow a fixed protocol that changes only in the event of unacceptable toxicity or unequivocal evidence of cancer progression.

There are three crucial elements that cancers and their treatment highly dyr amic. First, the distribution and abundance of $\mathrm{c}$ cer clones. These can be viewed as coe ing cyt types, with perhaps distinctive nic es and et al. 2016) that can chan, onidl Second, through feedbacks betwe cand r cell a tivities, the host and intratum al perties, the tumor ecosystem can rap a change size, character, and spatial heterogene. Third, the heritable traits of sor or all of cancer cell types may chans dir tly in response to therapy or in resp th chan, in the tumor environme $\mathrm{Tb}$ first wo dynamics describe the econ ai aramics of cancers and the tumor enviro. nent; the third describes the evolutionary dynamics of changing frequencies and heritable phenotypes of the cancer cytotypes themselves. For these reasons, cancers are highly dynamic systems with tremendous spatial and temporal heterogeneity that can rapidly change with perturbations such as applied therapy. Almost certainly, the tumor and its resident cancer-cell populations treated in the second cycle of chemotherapy are quite different that those treated in the first cycle. Indeed, any tumor that persists or recurs after therapy will differ radically from the tumor at diagnosis. The persistent or recurrent cancer cells will possess new phenotypes and genotypic profiles.

We propose that cancer therapy become as dynamic as the tumor system being treated. Ideally, treatment strategies should anticipate, corral, and stay ahead of the intratumoral evolution through strategic application of different drugs and drug doses that move beyond the traditional goal of maximal cell death. Maximum control of the tumor becomes the goal, in which the objective can include quality and longevity of life, cumulative dosing of therapies (radiation, chemo-agents, and/or immunotherapy), an the atient's sense of well-being both durir and in e absence of therapy.

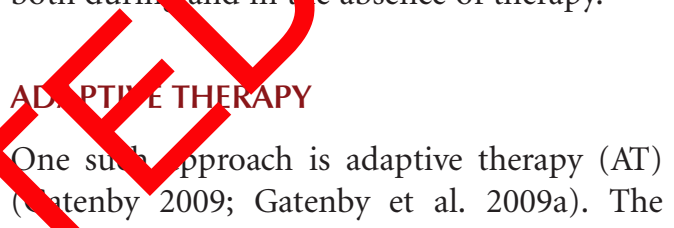
pren se of AT is similar to current pest management in that the time to progression of extant anticancer drugs can be revised if their administration is guided by Darwinian principles. This approach has a number of features that differ from conventional chemotherapy strategies. First, the goal of AT is maximizing progression-free survival and not abatement in cancer burden. Second, the amount of drug administered is the minimum necessary to maintain patient quality of life and tumor stability. Third, the drugs and dose schedules are not fixed but instead constantly adjusted to steer and exploit evolutionary dynamics and maintain a stable tumor.

Although there are a number of Darwinian dynamics that may be exploited during cancer therapy, the theory behind AT focuses on the phenotypic costs of the molecular mechanism(s) of resistance. Extant or potential cancer phenotypes within the ecological context of the tumor reside on an adaptive landscape describing fitness as a function of a cancer cell's phenotype. It is dependent on the fitness of that phenotype compared with that of other existent populations. Note that the fitness of any phenotype depends on context. A phenotype that, for example, expressing the PgP membrane pump is fitter than nonexpressing 
R. Gatenby and J. Brown

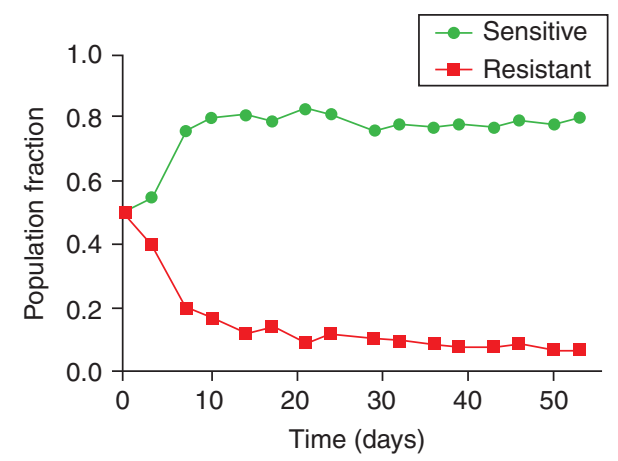

Figure 1. Illustrating the evolutionary consequence of the cost of resistance. To mimic growth dynamics of resistant and sensitive cell populations, labeled MCF7 (sensitive) and MCF7/Dox (resistant) cells were cocultured in the absence or doxorubicin with physiological levels of glucose. The phenotypic cost of resistance decreased fitness of the resistant cells and allowed the sensitive population to proliferate at the expense of the resistant phenotype.

cells in the presence of chemotherapy. Ho rever, in the absence of therapy, owing to the c t of the resistance mechanism, the fitn the sistant cells will be lower than that fo Ms re cells (Fig. 1). In this case, th on ress a cancer cell depends on its abilit to cr moete uccessfully with other cancen thin the context of the presence or 2 nce of 1 rapy. To exploit this, AT administers lin ited therapy with the explicit goal 1 maintaininga stable population of treatmo t-se oitive cells (Fig. 2). Therapy is redy or ithb once the tumor size is star azed Altho, on this may allow for some tun $r$ all prowferation, at the expense of the resista cells, the fitness advantage of sensitive cells will arlow their population to grow. Repeated administrations of small doses of drugs are then used to reduce the tumor volume. The goal is to administer "much less than maximum" dose while shrinking or maintaining at an acceptable level the overall tumor burden and total population size of cancer cells.

The success of AT is dependent on the cost of resistance and the competitive interaction between sensitive and resistant cancer cells. AT can be expected to be superior to maximum dose if three conditions are met. First, the therapy is highly effective against sensitive cancer cells. Second, sensitive cells outcompete resistant cells in the absence of therapy. Third, sensitive cells in the absence of therapy can proliferate faster than resistant cells can in the presence of therapy. This leads to either persistent or declining cycles of total tumor burden. Initial therapy rapidly drops the population size of sensitive cells. This results in the evolution and increase of resistant populations of cancer cells. Therapy is then stopped before the resistant cells completely replacing the sensitive cells. At this point, the resistant cells by virtue of their faster proliferation rates and greater competitive oill, will both increase and suppress the $\mathrm{r}$ istant ce . Before the sensitive cells retury $1 \mathrm{~g}$ to reate $\mathrm{ng}$ population sizes, therap must resu, This sequence represents con ate cy Such cycling works because of the the can decisively crash the sensitive $d$ 's, and the recovery of the sensitive cells prow de the therapy for the resistant cells. In practice, effective AT will require tailoring the therapy protocol to the nature of the interventions, the characteristics of the particular cancer, the nature and cost of resistance, useful metrics of tumor burden and composition, and associated mathematical model to integrate tumor metrics to determine and manage optimal timing of therapy dosing.

The AT hypothesis was initially framed using catastrophe theory (Gatenby and Frieden 2008). For any drug or drug combination, it can be shown that the probability of eradicating all of the tumor cells is maximized when phenotypes and environments are homogeneous. In this mathematical model, intratumoral heterogeneity results in phase differences between therapy and tumor that permits survival. The biological interpretation of "phase difference" is therapy that is unsuccessful because of the pretreatment presence of resistant phenotypes or environments that are relatively sheltered from the toxic effects of therapy and permit rapid evolution and proliferation of cancer cells with resistant strategies. It is not unsuccessful because of the evolution of resistance during therapy. Showing the feasibility of this theoretical model, a preclinical model of ovarian cancer (OVCAR-3 cells) was developed and treated 

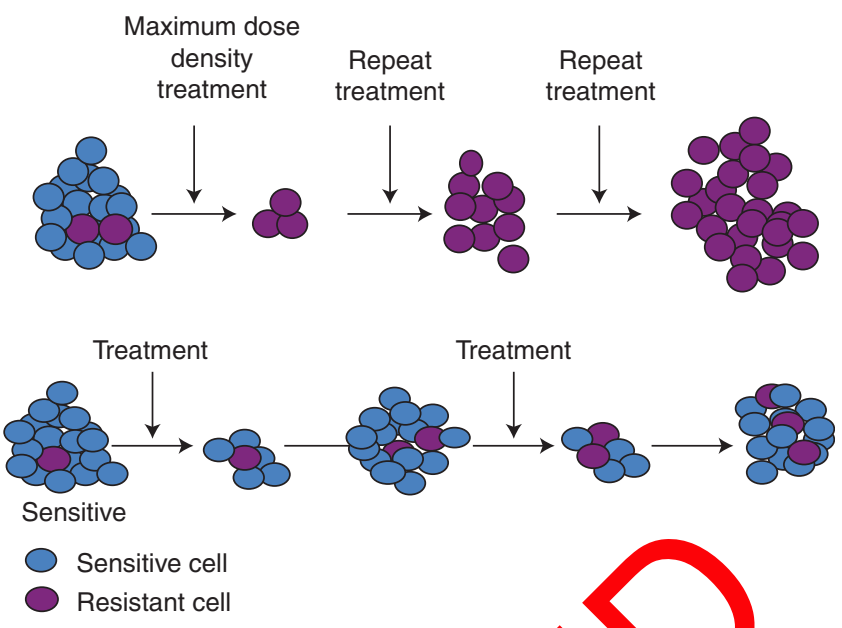

Figure 2. Conventional, high dose-density treatment compa a to an oly on-based strategy. (Top row) Conventional high dose-density therapy explicitly aims to eli anate cance cells that are sensitive to therapy. However, this maximally selects for resistant phenotypes $\mathrm{d}$ el nates $\mathrm{mpetitors} \mathrm{permitting} \mathrm{rapid} \mathrm{progres-}$ sion - an evolutionary dynamic termed "competitive ase." (Bo to row) Adaptive therapy (AT) explicitly aims to maintain a small population of cells that are sensit to treatment. Although the resistant cells survive, the metabolic cost of the molecular machinery resistance Fig. 1) renders them less fit in the absence of therapy. Thus, when therapy is withdrawn, the umor will regrow but the fitness advantage of the sensitive cells allows them to proliferate at the expense of the esistant $\mathrm{p}$ pulation. At the end of each cycle, the tumor remains sensitive to therapy.

with carboplatin. Control andar therapy $(60 \mathrm{mg} / \mathrm{kg} \mathrm{q} 4$ days $\mathrm{x} 3$ ) and $\delta$ gro ps were established. The AT a ,orn was based solely on tumor volume llowing he initial carboplatin dose of $50 \mathrm{mg} / \mathrm{h}_{\mathrm{c}}$ tumor volumes were measured ey $y$ three days. Subsequent doses of carboplati wer then adjusted to maintain stable or lume for example, if the tumor vol me it rrease in size for two consecutive mea yr nermo the dose of carboplatin would be inc ased. Similarly, doses would be decreased when tumor size declined. Compared with control and standard therapy, this AT resulted in prolonged tumor control and improved survival of mice (Gatenby et al. 2009a).

In these and in more recent studies using breast cancer cell lines, an unexpected observation is a biphasic pattern in tumor response to ATwas noted. That is, when treatment is initially applied, the tumors are typically growing exponentially. Forcing the growth curve to plateau required the full treatment dose. However, once the tumor volume stabilized the amount of drug necessary to maintain stability diminished rapid. In the above experiment, prolonged tumor control was often maintained using $5 \mathrm{mg} / \mathrm{kg}$ carboplatin. Of note, the computational models did not predict this phenomenon and it remains under investigation, but may be caused by tumor vascularity. Over time, it appeared that the tumor's blood system equilibrated on a more normal vascularity than the greatly dysregulated angiogenesis seen in the tumors of the control and standard of care mice. This observation points to the need to model and appreciate all three dynamics: cancer cell population size and evolution, tumor size and heterogeneity, and cancer cell-tumor ecosystem feedbacks.

\section{INCREASING THE COST OF RESISTANCE- ERSATZDROGES}

As mentioned previously, membrane extrusion pumps are a common mechanism of cancer cell resistance to chemotherapy. Targeting these pumps with treatments that reduces their effectiveness has been explored extensively but has 
not resulted in any clinically effective strategies to prolong treatment response (Gatenby et al. 2009b). As noted above, an alternative approach exploits the evolutionary consequences of the metabolic capital and operation costs for maintenance of the pumps. For example, Broxterman et al. (1988) showed that the extrusion activity of PgP pumps activated by verapamil could consume cell's ATP budget by 50\%. Importantly, in the intratumoral environment of limited resources, this increased ATP demand requires diversion of substrate from other activities including proliferation and invasion. Thus, an alternative treatment strategy to blocking pump function, seeks to exhaust the cancer cell's resources by maximizing pump activity through administration of nontoxic (or minimally toxic) substrate (Kam et al. 2015). Thus, by forcing cells to expend energy to extrude a "fake drug" (hence the name ersatzdroges) in the absence of chemotherapy, this strategy increases the pheno pro cost of the cells' resistance strategy.

Currently available ersatzdroges, incl ling antibiotics and verapamil (a calc an chant. blocker prescribed to treat arrhy mi, se used in the treatment of 9 , lisea s. Thus, when the tumor cell etect the resence of these "fake" drugs us as resistant mechanisms such as the "QR1 sys $m$ to pump the drug from the aytosol.

Exposur to verapand in a preclinical model of ast ancer dramatically altered the energy nan wi cancer cells and reduced bot' prol eratio and invasion. In vivo and in vitr cormmonation of various ersatzdroges signifi ntly increased glucose flux in tumors and reduced tumor growth (Kam et al. 2015). The use of the esatzdroges as chaff exploits ecological and evolutionary dynamics. The resistant cells waste energy bailing the ersatzdroges. The cell becomes less competitive relative to other cells with fewer pumps. And, evolution should now disfavor the membrane pumps or select for fewer pumps thus maintaining original drug efficacy. This represents a form of evolutionary therapy known as a "double bind therapy" (Gatenby et al. 2009b). A double bind therapy uses the cancer's adaptive response to one therapy (in this case a cytotoxin) to make another more effective (forced bailing of the ersatzdroges) and vice-versa.

\section{TURNING THE TABLES: TARGETING THE ADAPTIVE STRATEGIES}

In general, the application of evolutionary strategies to optimize tumor therapy requires "temporal" thinking. As shown in AT, the therapist must look beyond the immediate effects of treatment (i.e., tumor cell reduction). It requires anticipating and exploiting the longerterm ecological and evolutionary dynamics as new pheno ${ }^{+}$IC $p$ perties arise and increase among th cancer olls. Thinking ahead may identif the ppo cunities for double bind the apies Fig. (Gatenby et al. 2009b) or suc s gar its" (Maley et al. 2004). How often $\mathrm{ca}_{\mathrm{a}}$ se find a first line treatment to ina ce a phenotypic adaptation that is then more high susceptible to an especially chosen second line therapy?

A double-bind strategy was successfully applied to antibiotic treatment of Heliobacter pylori (Fuentes-Hernandez et al. 2015). In cancer treatment, Antonia et al. (2006) examined the efficacy of a 553 vaccine in patients $(n=29)$ with small cell lung cancer. Most patients elicited immune responses but only one partial response was observed. However, when the patients subsequently underwent chemotherapy, a response rate of $67 \%$ was observed (compared with the historic response rate of $<5 \%$ ); efficacy increased in those patients that had the greatest immune activation (Chiappori et al. 2010). We interpret this as an example of an evolutionary double bind in which the tumor cells' adaptive strategy to the immunotherapy rendered them more vulnerable to cytotoxic drugs.

Finally, the ideal double bind therapy creates an evolutionarily futile cycle in which cyclical application of the treatments matches precisely the pattern of evolution of resistance in the underlying cancer populations. In our clinical example, it is possible that this evolutionary cycle could have been completed by revaccinating the patients after chemotherapy, which may have selected for increased p53 expression. 


\section{EXPLOITING PROPERTIES OF COMPLEX DYNAMIC SYSTEMS FOR CANCER CONTROL}

All current cancer therapies act as biocides with various mechanisms for killing cancer cells. This is certainly reasonable but also inevitably produces Darwinian forces that promote resistance. An alternative approach targets environmental selection forces to alter the underlying evolutionary dynamics with the explicit goal of promoting a less proliferative or invasive tumor phenotype. In general, cancers can be viewed as open complex dynamical systems: "complex" because the tumor has many components, "dynamic" because the components interact and change over time (often nonlinearly), and "open" because it must freely interact with the host (for instance, all cancer cells derive their nutrients from the host). Traditional analysis of such systems emphasizes the challen - In predicting outcomes because of their nor inear dynamics and sensitivity to initial cond ions and/or slight perturbations of $\mathrm{cu}+$ conar tions (weather is probably the $\mathrm{mot}$ t ar complex dynamic system) exa iple, the famous "butterfly effect" , posit an in ct flapping its wings in Asi can ase a tornado in North America.

Importantly howe $r$, it has been noted that this esta rshed patter of complex systems to magnif son small perturbations can be exploit to eer ty system along a desired coy a w th malmal application of force $\left(W_{a}\right.$ ar a 2$)$. This, of course, requires a modes understanding of the underlying intratumoral cynamics, but it does not require a full understanding. One has a sufficient level of understanding when interventions suggest themselves that perturb the tumor environment in ways that dissipate the cancer system or at least coax it along a less clinically aggressive path. What small, yet decisive, biological forces are available? Perturbing $\mathrm{pH}$ may prove useful. Most tumors are net-producers of acid and intratumoral acidosis has been shown to select for phenotypes that are highly motile and invasive. However, small perturbations of the extracellular $\mathrm{pH}$ (an increase of $\sim 0.2 \mathrm{pH}$ units) can alter these Darwinian dynamics and select for less aggressive, more indolent phenotypes (Ibrahim-Hashim et al. 2012).

\section{CLINICAL APPLICATIONS}

Mastering complex systems (as in weather forecasting) (Wang et al. 2012) require three components: (1) defining and mathematically framing first principles; (2) necessary and sufficient data to parameterize mathematical models; and (3) sophisticated computational methods.

We propose that evolutionary and ecological dynamis sern sirst principles in cancer therapy a that $c$ mputational models can be rea Aly co truct d (e.g., computer models for est $y$ mage ent are widely available). The ose of us le data is the greatest stumbling block to $b$ clinical application of evolutionary p nciples to cancer therapy. Although concerns for valing with "big data" from molecular analysis are often expressed in oncology, these data lack spatial and temporal resolution and therefore have limited value in evolutionary models. In fact, good time-series data in oncology is largely limited to serum markers and repeated cross-sectional imaging. There is an urgent need to develop methods that can use sparse data for computational models and extract more information from available clinical data (e.g., radiomic analysis of computed tomography [CT] and magnetic resonance imaging [MRI] images [Gatenby et al. 2013]).

Limitations withstanding, some clinical trials that illustrate successful application of evolutionary dynamics have been and are being performed (Schweizer et al. 2015). We suggest that the applications of evolutionary concepts and therapies can allow us make quantum strides by simply changing our use of existing therapies, patient and tumor metrics, and data analysis and modeling. Such results may be possible at a fraction of the cost of novel drug discovery and development.

\section{SUMMARY AND FUTURE DIRECTIONS}

Cancers are complex, dynamic systems that begin to evolve resistance strategies immediately 
R. Gatenby and J. Brown

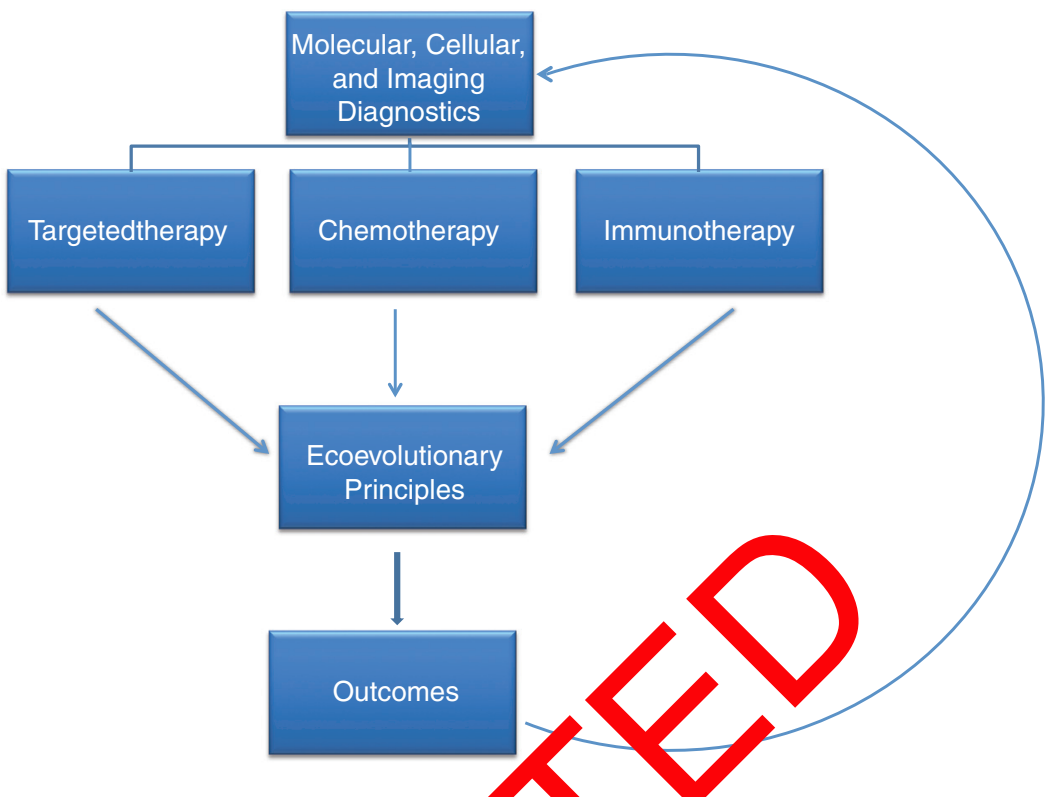

Figure 3. Current "personalized medicine" paradigms almo exclusive focus on defining predictive biomarkers that can identify effective treatments. This ap howh, howe fails to recognize that even highly effective therapies are almost always defeated by evolv ion of recistance. Here, we propose that "precision medicine" in cancer care requires both identification of 0 timal tre dynamics that govern response and resictance to herar and, thus, ultimate patient outcomes. ment modality and understanding of the Darwinian

on the application of hy th arapy. Current cancer treatment is ty ica appliec in a static fashion: the same ags, dos and schedules are administered until e protocol ends or the tumor prog sses. When he cancer cells of a tumor ress nd treatment, the typical strategy is to si $y$ g $m g$ of the same. In contrast, evg ation pased nerapy seeks to become as ada iv anumexible as the tumor populations being ated. This will require a new paradigm in eancer therapy in which Darwinian dynamics are explicitly incorporated into trial design and execution (Fig. 3). In AT, for example, once the tumor response to a specific treatment is observed, the best course might be to switch to a new strategy or withdraw therapy because ongoing treatment with the successful drug will only result in greater selection for resistance.

In preclinical experiments (Gatenby et al. 2009a; Silva et al. 2012; Kam et al. 2015), we have shown that applying evolutionary principles to conventional chemotherapy agents can substantially prolong progression-free survival in both breast and ovarian cancer. Schweizer et al. (2015) recently showed that evolutionary principles could be used to prolong response to anti-androgen therapy in a cohort of men with castrate-resistant prostate cancer. A clinical trial using an adaptive-therapy algorithm for abiraterone therapy in men with castrate-resistant prostate cancer has recently opened and appears superior to continuous therapy.

There are difficulties in clinical application. These include the requirement to collect reliable time-series data allowing the internal evolutionary dynamics of the cancer to be observed, measured, and estimated. In fact, repeated measures of changes in tumors over time and space are typically quite sparse, largely limited to serum markers and clinical imaging. Future directions must focus on converting clinical data into a dynamical understanding of the complex environmental and phenotypic changes that drives tumor evolution in response to therapy. Ultimately, this understanding will likely re- 
quire sophisticated patient-specific computational models to provide treating physicians with decision support tools to optimize cancer therapy.

\section{ACKNOWLEDGMENTS}

This work is supported by National Institutes of Health (NIH) Grants U54CA143970-1 and RO1CA170595 and a grant from the James S. McDonnell Foundation.

\section{REFERENCES}

Anderson AR, Quaranta V. 2008. Integrative mathematical oncology. Nat Rev Cancer 8: 227-234.

Antonia SJ, Mirza N, Fricke I, Chiappori A, Thompson P, Williams N, Bepler G, Simon G, Janssen W, et al. 2006. Combination of p53 cancer vaccine with chemotherapy in patients with extensive stage small cell lung cancer Clin Cancer Res 12: 878-887.

Axtell RC, Arends JJ. 1990. Ecology and mana ement of arthropod pests of poultry. Annu Rev Ento l 35: $101-126$.

Ayala FJ, Dobzhansky T. 1977. "Nothing in iolo makes sense except in the light of evolution": The do as Duzhansky: 1900-1975. J Hered 68

Broxterman HJ, Pinedo HM cuiper CM, K tein LC, Schuurhuis GJ, Lankelmat 1) I a acum y verapamil of a rapid increase in $\mathrm{P}$ con pption in multidrugresistant tumor cell a EB J 2: 2 $2-2282$.

Chiappori AA, Saliman A, Janssen WE, Antonia SJ, Gabrilovich D 2010. INGN 25: A dendritic cell-based p53 vacci (Ad. 3-DC) in small cell lung cancer: Observed a ci on betreen immune response and enhanc expert Opin Biol Ther 10: 98 - 991.

Oxnard GR, Hutchinson K, Ohashi K, Son ar R, Wang L, Amato KR, Arcila M, Sos ML, et al. 2011. vtimization of dosing for EGFR-mutant nonsmall celMung cancer with evolutionary cancer modeling. Sci Transl Med 3: 90ra59.

Easwaran H, Tsai HC, Baylin SB. 2014. Cancer epigenetics: Tumor heterogeneity, plasticity of stem-like states, and drug resistance. Mol Cell 54: 716-727.

Fletcher JI, Haber M, Henderson MJ, Norris MD. 2010. ABC transporters in cancer: More than just drug efflux pumps. Nat Rev Cancer 10: 147-156.

Fuentes-Hernandez A, Plucain J, Gori F, Pena-Miller R, Reding C, Jansen G, Schulenburg H, Gudelj I, Beardmore R. 2015. Using a sequential regimen to eliminate bacteria at sublethal antibiotic dosages. PLoS Biol 13: e1002104.

Gatenby RA. 2009. A change of strategy in the war on cancer. Nature 459: 508-509.

Gatenby RA, Frieden BR. 2008. Inducing catastrophe in malignant growth. Math Med Biol 25: 267-283.
Gatenby RA, Silva AS, Gillies RJ, Frieden BR. 2009a. Adaptive therapy. Cancer Res 69: 4894-4903.

Gatenby RA, Brown J, Vincent T. 2009b. Lessons from applied ecology: Cancer control using an evolutionary double bind. Cancer Res 69: 7499-7502.

Gatenby RA, Grove O, Gillies RJ. 2013. Quantitative imaging in cancer evolution and ecology. Radiology 269: 8-15.

Gnoni A, Silvestris N, Licchetta A, Santini D, Scartozzi M, Ria R, Pisconti S, Petrelli F, Vacca A, Lorusso V. 2015. Metronomic chemotherapy from rationale to clinical studies: A dream or reality? Crit Rev Oncol Hematol 95: 46-61.

Ibrahim-Hashim A, Cornnell HH, Abrahams D, Lloyd M, Bui M, Gillies RJ, Gatenby RA. 2012. Systemic buffers inhibit carcinogenesis in TRAMP mice. J Urol 188: 624-631.

Jansen G, Gate 1 tipis CA. 2015. Opinion: Control vs. eradic on: Appl, ng infectious disease treatment strategies to incer. Pro Natl Acad Sci 112: 937-938.

KamY as T, Tian Fo atan P, RuizE, Martinez G, Minton Gillies, Gate, RA. 2015. Sweat but no gain: Inhibit. diferatin of multidrug resistant cancer cells with "ersa droge Int J Cancer 136: E188-E196.

oyd MC, anningham JJ, Bui MM, Gillies RJ, Brown JS, atenby RA. 2016. Darwinian dynamics of intratumoral ho rogeneity: Not solely random mutations but also variable environmental selection forces. Cancer Res 76: 3136-3144.

Maley CC, Reid BJ, Forrest S. 2004. Cancer prevention strategies that address the evolutionary dynamics of neoplastic cells: Simulating benign cell boosters and selection for chemosensitivity. Cancer Epidemiol Biomarkers Prev 13: 1375-1384.

Meads MB, Gatenby RA, Dalton WS. 2009. Environmentmediated drug resistance: A major contributor to minimal residual disease. Nat Rev Cancer 9: 665-674.

Moulder S. 2010. Intrinsic resistance to chemotherapy in breast cancer. Womens Health 6: 821-830.

Neve P, Vila-Aiub M, Roux F. 2009. Evolutionary-thinking in agricultural weed management. New Phytologist 184: $783-793$.

Norton L, Simon R. 1986. The Norton-Simon hypothesis revisited. Cancer Treat Rep 70: 163-169.

Oliveira EE, Guedes RNC, Totola MR, De Marco P Jr. 2007. Competition between insecticide-susceptible and -resistant populations of the maize weevil, Sitophilus zeamais. Chemosphere 69: 17-24.

Renton M, Busi R, Neve P, Thornby D, Vila-Aiub M. 2014. Herbicide resistance modelling: Past, present and future. Pest Manag Sci 70: 1394-1404.

Rodrigues DS, de Arruda Mancera PF. 2013. Mathematical analysis and simulations involving chemotherapy and surgery on large human tumours under a suitable cellkill functional response. Math Biosci Eng 10: 221-234.

Schneider J, Bak M, Efferth T, Kaufmann M, Mattern J, Volm M. 1989. P-glycoprotein expression in treated and untreated human breast cancer. Br J Cancer 60: 815-818.

Schweizer MT, Antonarakis ES, Wang H, Ajiboye AS, Spitz A, Cao H, Luo J, Haffner MC, Yegnasubramanian S, Carducci MA, et al. 2015. Effect of bipolar androgen therapy for asymptomatic men with castration-resistant prostate 
R. Gatenby and J. Brown

cancer: Results from a pilot clinical study. Sci Transl Med 7: 269ra2.

Silva AS, Kam Y, Khin ZP, Minton SE, Gillies RJ, Gatenby RA. 2012. Evolutionary approaches to prolong progression-free survival in breast cancer. Cancer Res 72: 63626370.

Silva R, Vilas-Boas V, Carmo H, Dinis-Oliveira RJ, Carvalho F, de Lourdes Bastos M, Remião F. 2015. Modulation of Pglycoprotein efflux pump: Induction and activation as a therapeutic strategy. Pharmacol Ther 149: 1-123.
Thews O, Nowak M, Sauvant C, Gekle M. 2011. Hypoxiainduced extracellular acidosis increases p-glycoprotein activity and chemoresistance in tumors in vivo via $\mathrm{p} 38$ signaling pathway. Adv Exp Med Biol 701: 115-122.

Wang WX, Ni X, Lai YC, Grebogi C. 2012. Optimizing controllability of complex networks by minimum structural perturbations. Phys Rev E 85: 026115.

Wind NS, Holen I. 2011. Multidrug resistance in breast cancer: From in vitro models to clinical studies. Int $J$ Breast Cancer 2011: 967419.

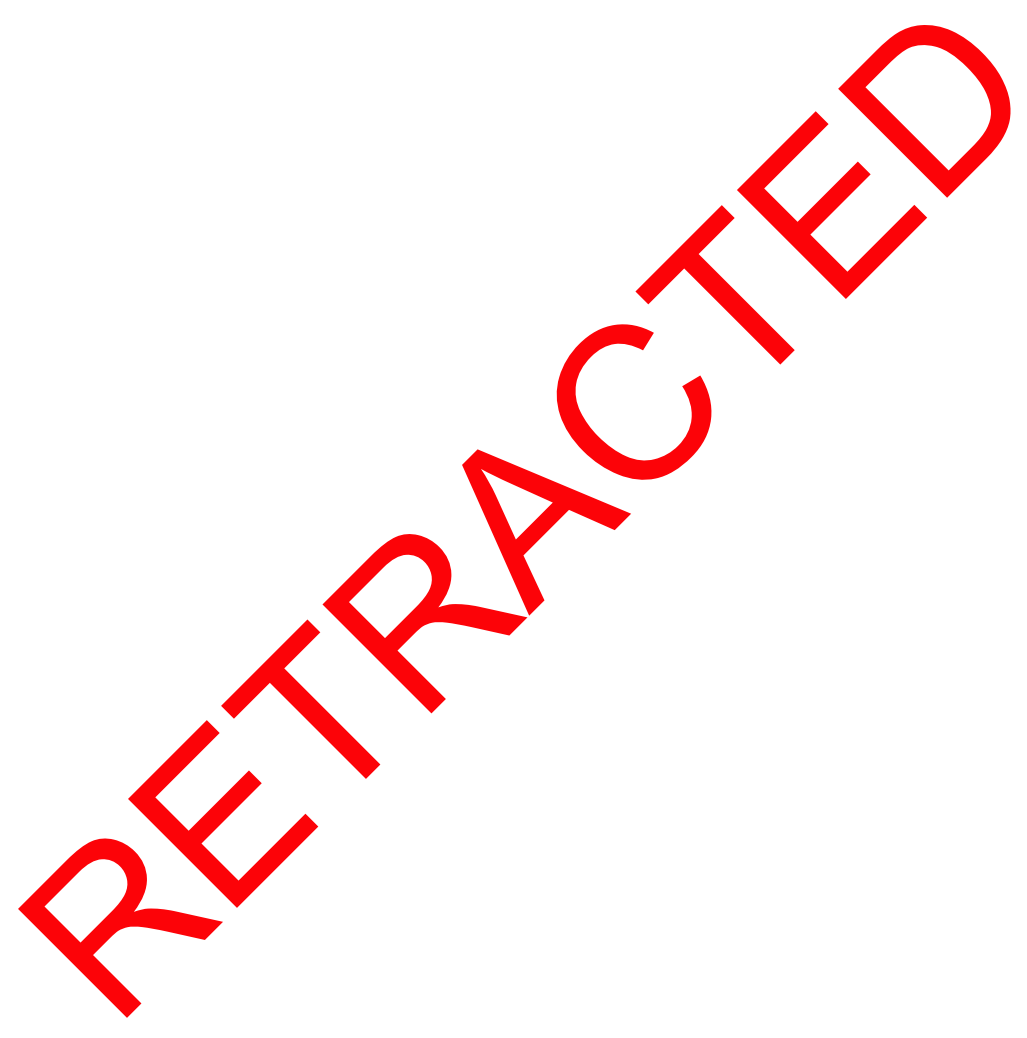




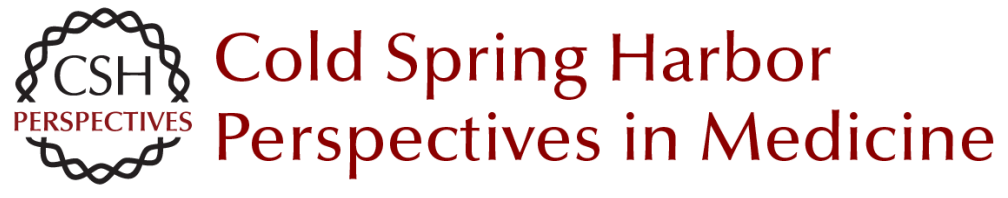

\section{The Evolution and Ecology of Resistance in Cancer Therapy}

Robert Gatenby and Joel Brown

Cold Spring Harb Perspect Med 2018; doi: 10.1101/cshperspect.a033415 originally published online July 14,2017

\section{Subject Collection Cancer Evolution}

The Evolution and Ecology of Resistance in Cancer Therapy

Robert A. Gatenby and Joel S. Brown

Big Bang Tumor Growth and Clonal Evolution Ruping Sun, Zheng Hu and Christina Curtis

Observing Clonal Dynamics across

Spatiotemporal Axes: A Prelude to Quantitative

Fitness Models for Cancer

Andrew W. McPherson, Fong Chun Chan and

Sohrab P. Shah

Evolution of Premalignant Disease

Kit Curtius, Nicholas A. Wright and Trevor A. Graham

The Role of Aneuploidy in Cancer Evolution Laurent Sansregret and Charles Swanton

Treatment-Induced Mutagenesis and Selective Pressures Sculpt Cancer Evolution

Subramanian Venkatesan, Charles Swanton, Barry S. Taylor, et al.

Chromosomal Instability as a Driver of Tumor Heterogeneity and Evolution Samuel F. Bakhoum and Dan Avi Landau

Coevolution of Leukemia and Host Immune Cells

in Chronic Lymphocytic Leukemia

Noelia Purroy and Catherine J. Wu
The Evolution and Ecology of Resistance in

Cancer Therapy

Robert Gatenby and Joel Brown

Phylogenetic Quantification of Intratumor

Heterogeneity

Thomas B.K. Watkins and Roland F. Schwarz

The "Achilles' Heel" of Cancer and Its Implications for the Development of Novel Immunotherapeutic Strategies

Kroopa Joshi, Benjamin M. Chain, Karl S. Peggs, et al.

Homeostasis Back and Forth: An Ecoevolutionary

Perspective of Cancer David Basanta and Alexander R.A. Anderson

Principles of Reconstructing the Subclonal

Architecture of Cancers

Stefan C. Dentro, David C. Wedge and Peter Van LoO

Tumor Microenvironment and Differential

Responses to Therapy

Eishu Hirata and Erik Sahai

Order Matters: The Order of Somatic Mutations Influences Cancer Evolution

David G. Kent and Anthony R. Green

The Cellular Origin and Evolution of Breast

Cancer

Mei Zhang, Adrian V. Lee and Jeffrey M. Rosen

For additional articles in this collection, see http://perspectivesinmedicine.cshlp.org/cgi/collection/ 